\title{
3D printed models of congenital heart disease: How accurate and how useful
}

\section{are they?}

\author{
Ivan Lau, ${ }^{1}$ Andrew Squelch, ${ }^{2,3}$ and Zhonghua Sun ${ }^{1}$
}

1. Discipline of Medical Radiation Sciences, School of Molecular and Life Sciences, Curtin University, Perth, Western Australia, Australia

2. Discipline of Exploration Geophysics, Western Australian School of Mines, Minerals: Energy and Chemical Engineering, Curtin University, Perth, Western Australia, Australia

3. Computational Image Analysis Group, Curtin Institute for Computation, Curtin University, Perth, Western Australia, Australia

\section{Editorial}

Please cite this paper as: Lau I, Squelch A and Sun Z. 3D printed models of congenital heart disease: How accurate and how useful are they? AMJ 2019;12(11):312-314.

https://doi.org/10.35841/1836-1935.12.11.312-314

\section{Corresponding Author:}

Prof Zhonghua Sun

Discipline of Medical Radiation Sciences, School of Molecular and Life Sciences, Curtin University, Perth, WA, 6845, Australia Email: z.sun@curtin.edu.au

\section{ABSTRACT}

Three-dimensional (3D) printing in the domain of congenital heart disease (CHD) is still in its infancy. The aim of this editorial is to highlight the key findings of a recently published systematic review and meta-analysis on the accuracy and clinical value of 3D printed heart models (3DPHM). The analysis found that 3DPHM can be generated with high accuracy and the most reported application of 3DPHM is to facilitate pre-operative planning.

\section{Key Words}

3D printing, additive manufacturing, stereolithography, 3D model, congenital heart disease, congenital heart defect

\section{Introduction}

Three-dimensional (3D) printing has been widely utilized in different specialties within the medical field for decades. ${ }^{1-3}$ However, in the domain of cardiovascular specialties, this technology is still considered fairly new. ${ }^{4}$ The conventional way to interpret the medical images from twodimensional (2D) flat screen lacks comprehensiveness, hence tangible 3D printed heart models (3DPHM) were created to improve the users' perception on the depth information of the cardiac anatomies. ${ }^{3-6}$ Although there are increasing reports on the use of 3DPHM, most of them remain anecdotal, and very few of them perform quantitative measurements of the accuracy and clinical value of the 3DPHM. ${ }^{4,7}$ The relevant questions are: if the 3DPHM are accurate, to what extent are they accurate; if the 3DPHM are useful, in what areas are they useful; and do all the studies share the same findings? This editorial aims to provide a succinct summary of a recently published systematic review and meta-analysis on the accuracy and clinical value of 3DPHM. ${ }^{4}$

How accurate and useful are the 3D printed heart models?

A total of 24 articles were included in the systematic review and 7 of them were used in the meta-analysis. ${ }^{4}$ Based on the findings of this review, there are 4 different imaging modalities that can be used to generate 3DPHM, with computed tomography angiography (CTA) being the dominant, followed by cardiac magnetic resonance (CMR), echocardiography, and rotational angiography. Materialise Mimics is the most popular software for cardiac image segmentation, with 12 out of 24 studies (50 per cent) reporting its application. ${ }^{4}$

It was found that the accuracy of the 3DPHM is reported in relatively few studies since only 7 out of 24 provided the statistical measurements of the 3DPHM. Nevertheless, all of these studies shared the same findings: 3DPHM is highly accurate. Based on the meta-analysis of 3 eligible studies, the pooled mean deviation of 3DPHM measurement and original medical images measurement is $0.04 \mathrm{~mm}, 95$ per cent $\mathrm{Cl}(-0.16,0.23)$ (Figure 1$)$, which is 
considered negligible as it is below the image resolution of routine medical CTA and CMR images. This however, needs to be interpreted with care as the Cochran's $Q$ test demonstrates high heterogeneity among the studies $(p=0.0468){ }^{4}$ It is also important to note that the quantitative synthesis of 3DPHM accuracy did not take into account the 3D printing technique and segmentation software used in the individual studies, which could also explain the significant heterogeneity among the studies.

In terms of the uses of 3DPHM, the most reported use of 3DPHM is its role in facilitating pre-operative planning, followed by medical education, communication, pre-surgical simulation, and intra-operative orientation. ${ }^{4}$ Meta-analysis is only possible for 4 out of 12 studies which reported the use of $3 \mathrm{DPHM}$ in medical education. It was found that the 3DPHM group scored less in the test group compared to the control group, although it did not reach statistical significance $(-0.43$, 95 per cent $\mathrm{Cl}(-4.75,3.88), \mathrm{p}=0.844)$ (Figure 2). This finding also needs to be interpreted carefully as the Cochran's $Q$ test demonstrates high variations among the studies $(p<0.001){ }^{4}$ Although the use of 3DPHM might not improve the students' short-term knowledge on CHD (measured by the test scores), the students' learning experience and satisfaction were reported to be improved in all 12 studies. ${ }^{4}$

The 3DPHM were also found to be valuable in helping surgeons to decide and define the best surgical approach, particularly for complex CHD. 3DPHM also have the potential to reduce the operational cost of the surgery due to the reduction in surgical duration, however this needs to be investigated further with a comprehensive cost-benefit analysis. ${ }^{4}$ The role of $3 \mathrm{DPHM}$ in improving communication within clinical practice is uncertain. Some studies reported that the use of 3DPHM can enhance patient-doctor interaction, however it does not seem to shorten the consultation duration, nor does it increase the short-term parental knowledge on CHD. Despite the contradicting results, 3DPHM is perceived as a complementary tool in patientdoctor communication, in addition to the original medical images. $^{4}$

\section{Conclusion}

Based on the results from this systematic review and metaanalysis, it can be concluded that there is a paucity of comprehensive and systematic studies about 3D printing of $\mathrm{CHD}$ in the current literature. Although $3 \mathrm{D}$ printing of $\mathrm{CHD}$ is still considered at its early development stage, the results from current studies are promising. 3DPHM can be fabricated with high accuracy, and multiple groups of stakeholders can benefit from the application of this technology in the diagnosis and treatment of CHD. However, more studies based on larger sample sizes are required to validate these positive findings. Future studies should also focus on investigating the cost-benefit of implementing 3D printing technology in the domain of $\mathrm{CHD}$ before it is incorporated into a routine diagnostic approach.

\section{References}

1. Kim GB, Lee $\mathrm{S}$, Kim $\mathrm{H}$, et al. Three-Dimensional Printing: Basic Principles and Applications in Medicine and Radiology. Korean J Radiol. 2016;17(2):182-197.

2. Batteux C, Haidar MA, Bonnet D. 3D-Printed Models for Surgical Planning in Complex Congenital Heart Diseases: A Systematic Review. Front Pediatr. 2019;7.

3. Shi D, Kai L, Zhang $X$, et al. Applications of threedimensional printing technology in the cardiovascular field. Intern Emerg Med. 2015;10:769-780.

4. Lau I, Sun Z. Dimensional Accuracy and Clinical Value of 3D Printed Models in Congenital Heart Disease: A Systematic Review and Meta-Analysis. J Clin Med. 2019;8:1483.

5. Biglino G, Koniordou D, Gasparini M, et al. Piloting the Use of Patient-Specific Cardiac Models as a Novel Tool to Facilitate Communication During Clinical Consultations. Pediatr Cardiol. 2017;38:813-818.

6. Valverde I, Gomez-Ciriza G, Hussain T, et al. Threedimensional printed models for surgical planning of complex congenital heart defects: An international multicentre study. Eur J Cardiothorac Surg. 2017;52:1139-1148.

7. Sun Z, Lau I, Wong YH, et al. Personalized threedimensional printed models in congenital heart disease. J Clin Med. 2019;8:522.

\section{PEER REVIEW}

Peer reviewed.

\section{CONFLICTS OF INTEREST}

The authors declare that they have no competing interests.

\section{FUNDING}

None 
Figure 1: Forest plot for mean bias of the 3DPHM

measurement and the digital images measurement. 3D,

three-dimensional; B-A, Bland-Altman; MRAW, raw mean difference; $\mathrm{Cl}$, confidence interval; $\mathrm{SE}$, standard error. Reprinted with permission under the open access from Lau and Sun. ${ }^{4}$

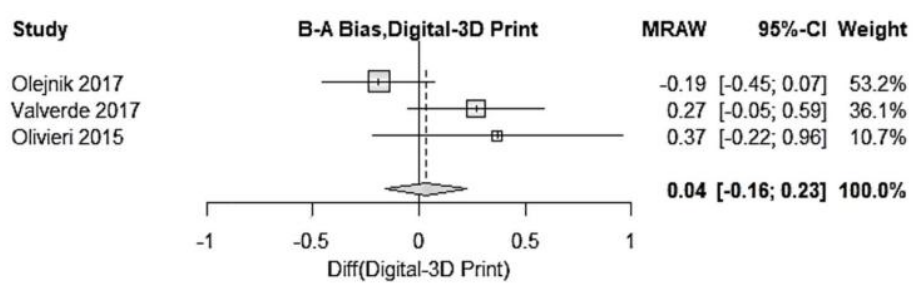

Fixed effect model

Heterogeneity: $I^{2}=67 \%, \tau^{2}=0.0689, p=0.05$

Cochran's Q test: $\chi^{2}=6.12(p=0.0468)$

Bartlett's test: $\chi^{2}=4.8233(p=0.0897)$

Figure 2: Forest plot for mean differences in test scores between the 3DPHM and the control groups. 3D, threedimensional; $\mathrm{Cl}$, confidence interval; $\mathrm{MD}$, mean difference; SD, standard deviation; SE, standard error. Reprinted with permission under the open access from Lau and Sun. ${ }^{4}$

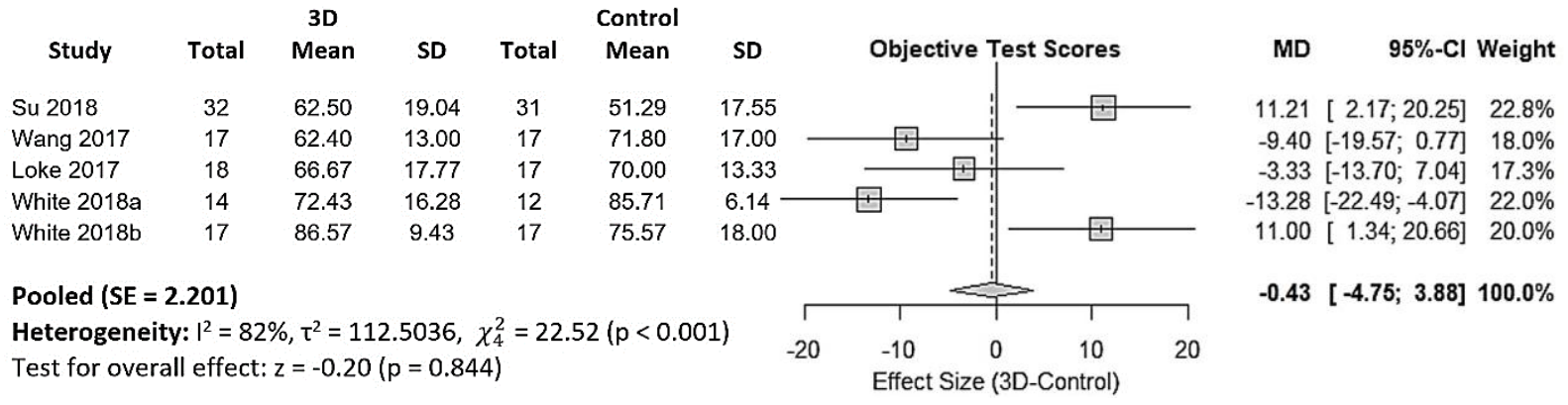

